\title{
Postcategorical filtering in a bar-probe task
}

\author{
A. H. C. VAN DER HEIJDEN \\ University of Leiden, Leiden, The Netherlands
}

\begin{abstract}
To investigate the causes of errors in partial-report bar-probe tasks, a set of (color) stimuli that allowed the use of a new method of analysis was devised. With these stimuli and this method of analysis, two important results were obtained. First, the number of location errors (i.e., errors consisting of naming the color of an item in a nonindicated position) was far below chance level with short probe delays and moved in the direction of chance level with increasing probe delays. Second, the number of genuine visual confusions first increased and then somewhat decreased with increasing probe delays. The results make it possible to choose among three current models of information processing for this type of task. The "orthodox" account and the "dual-buffer" account are not consistent with the data obtained. Postcategorical filtering, that is, the selection of identified information by using precategorical information, is compatible with the results reported.
\end{abstract}

In a partial-report bar-probe task, subjects are shown linear or circular arrays of elements (such as letters, digits, or forms) and a barmarker (probe) indicating which element is to be reported. The most important observation is that subjects can name the indicated element rather accurately (i.e., the proportion of correct reports is far above chance level) when the probe follows the display within an interval up to about $300 \mathrm{msec}$, under conditions that prevent eye movements in the direction of the target. A second observation is that accuracy of report declines gradually with increasing probe delays (see, e.g., Averbach \& Coriell, 1961). The present study is concerned with the problem of how we are to explain, or model, this pattern of results. A number of accounts have been proposed for these results. The basic questions the models address concern how to explain subjects' accurate performance and how to explain the decline in accuracy with increasing probe delay. We briefly describe three of these models, one precategorical selection model and two postcategorical selection models.

In the most common view, it is held that a representation of a brief visual display is stored initially for a short period of time after exposure, in a precategorical visual memory (variously named visual information store, iconic memory, sensory register, etc.). This mem. ory is characterized by high capacity and rapid decay. To be reported, items have to be rescued from this

I am grateful to Hanneke van Kempen, Mark Neerincx, and Herman Kruysse for conducting the experiments, analyzing the data, and providing valuable comments and criticisms during nearly all stages of this research. I thank $H$. Werner for assisting in analyzing C. W. Eriksen and Rohrbaugh's (1970) formula for determining chance expectancy, and D. Mewhort for providing valuable suggestions for the improvement of the manuscript. My mailing address is: Department of Psychology, Unit of Experimental Psychology, University of Leiden, Hooigracht 15, 2312 KM Leiden, The Netherlands. memory; that is, the items have to be transferred from this store to another store in which decay is negligible. It is assumed that this transfer process is selective, in the sense that priority can be given to one or more required items, if these items are appropriately indicated or marked. The barmarker appears to be an appropriate indicator, and, therefore, the rather high accuracy is explained. However, the success of the transfer process depends on the integrity of the representation in the visual memory. The more the probe is delayed, the more the representation will decay. Therefore, a decline in accuracy with increasing probe delay will be observed. This is a precategorical selection model because it is assumed that unidentified information is selected for further processing (i.e., transfer).

Van der Heijden (1981) pointed out that the foregoing view is at variance with another body of relevant evidence. In selective attention tasks in which latency, instead of accuracy, is used as the main dependent variable, it is easy to show that identification is nonselective and automatic (see, e.g., B. A. Eriksen \& C. W. Eriksen, 1974, C. W. Eriksen \& Hoffman, 1973, and Gatti \& Egeth, 1978). To explain such results, and the results obtained in partial-report bar-probe tasks, Van der Heijden proposed a postcategorical selection model, that is, a model postulating that identified information is selected for report. Automatic, nonselective processing of the information is accounted for by postulating a system of counters or logogens: transducers in which sensory information activates "programs of motor instructions." Upon presentation of a multielement stimulus array, a number of logogens is activated briefly; that is, sensory features and activated programs are simultaneously present in the system as linked or con nected units. A selective operation, postcategorical filtering, operating at the sensory level, selects the appropriate logogen, thereby selecting the correct program. ${ }^{1}$ As in the precategorical selection model, the 
selective operation explains the rather high accuracy in partial-report bar-probe tasks. The assumption that (at least) sensory information (i.e., the activation at the input side of the logogens) decays rapidly over time is sufficient to explain the declining accuracy with increased probe delays.

Mewhort, Campbell, Marchetti, and Campbell (1981) emphasized that the error data obtained in bar-probe experiments are inconsistent with a precategorical selection model. The increase in errors associated with increasing probe delay consists almost entirely of an increase in location errors (a report of a nonindicated letter from the display), whereas intrusion errors (a report of an item not on the display) show only a small increase (see, e.g., di Lollo, 1978, Mewhort et al., 1981, and Townsend, 1973). If the incorrect responses result from identifying a degraded sensory representation, a large increase in the number of intrusions should have occurred, and there would be no reason to expect that the errors would consist of location errors. The dissociation of the effects of probe delay on types of errors is consistent with the view that location information is rapidly lost while identity information is retained. Mewhort et al. (1981) proposed a "dual-buffer" model to account for the results. It is assumed that a representation of the stimulus first enters an unlimited capacity, precategorical sensory store, the feature buffer. An identifier, operating in parallel on all the information in this buffer, identifies the items and stores abstract representations of the items along with location information in a second buffer, the character buffer. It is assumed that the location information disappears relatively rapidly, while the identity information is retained. An attentional mechanism translates the abstract codes into a code suitable for output. This attentional mechanism, operating upon the identity and location information in the character buffer, ensures a rather high level of accuracy with short probe delays. The assumption that the relevant location information decays rapidly accounts for the decrease in accuracy with increasing probe delays. The assumption that identity information is retained is used to explain the fact that location errors and not intrusions increase with increasing probe delays.

The three models correspond in postulating (at least) two stores (separate levels of representation): a precategorical store containing unidentified visual informa. tion, a level of representation containing visual information (store A), and a postcategorical store containing identified information, a representation level containing categorized information (store B).

The models differ with regard to a number of issues. For present purposes, the most important differences concern (1) the level at which selective attention is thought to operate and (2) the nature of the items that are selected.

The first two models agree in that selective attention operates in or uses information in store A. Both models have to predict, therefore, that decay of information in this store will be reflected in performance. The last model assumes that selective attention operates in store B and predicts, therefore, that no evidence for decay of information in store A will show up in the data.

The last two models agree in that selective attention selects from among identified or categorized information (via sensory features in the visual domain and with location information in the character buffer, respectively). These two models have to predict that effects of identified irrelevant information can show up in the data. The first model assumes that selective attention selects from among unidentified information, and therefore there is no reason to expect that effects of identified irrelevant information will be reflected in the data.

So, before we can decide among the three models, we need information about (1) the effects of decaying information in store A and (2) the effects of identified irrelevant information on the results obtained in partialreport bar-probe tasks. Probe tasks, as presently used, do not provide this information, however. Responses are classified as correct reports, intrusion errors, or location errors. As Mewhort et al. (1981, Note 2) correctly pointed out, this classification is a straightforward descriptive classification of responses, but it says nothing about the cause of the responses. A location error, for instance, can result from pure guessing, from a failure of identification, and from a failure of localization; an intrusion error can result from pure guessing, a failure of identification, and even from a failure of localization combined with a failure of identification. Such a coarse descriptive categorization of responses cannot provide the information we need for deciding among the three models outlined above. We need a further breakdown of responses according to their cause.

In the present study, I shall report a first step in the further analysis of responses (some problems we encountered will be briefly hinted at later in this study). The General Method describes the paradigm and the method of analysis. The General Discussion describes the implications for the three models of the results obtained in the two experiments reported in this study.

\section{GENERAL METHOD}

Items, displays, and method of analysis were selected and developed simultaneously in a number of pilot studies. For explanatory purposes, here we describe first the items used, then the composition of the displays, and then the method of analysis as used in the main part of our study.

\section{Stimulus Items}

In this study, we needed four pairs of items, chosen in such a way that visual confusions (i.e., genuine misidentifications of an item) resulted mainly in the naming of the other member of the pair and virtually never in the naming of a member of another pair. We tried several kinds of items, and at the end came up with four pairs of colored disks. These colors were: light and dark red, light and dark biue, light and dark green, and light and dark gray. (Within pairs, there were not only brightness differences. but also hue and saturation differences.) 
Pilot work showed that, after some practice, with $100-\mathrm{msec}$ tachistoscopic presentations of single items, virtually all errors consisted of the name of the other member of the pair. Only with very short exposures (less than $10 \mathrm{msec}$ ) did some errors consisting of naming a member of another pair occur. The choice of colors as stimulus items and the results of our pilot work seem to assure that genuine visual confusions (i.e., real misidentifications) nearly always result in within-pair errors. Only when misidentifications approach or equal random guessing do between-pair errors result. (Future research will have to be concerned with finding other and possibly better sets of stimuli that fulfill the conditions specified above.)

\section{Displays}

For the main experiment, 48 five-element displays were constructed. The five colored disks (diameter $=.4 \mathrm{deg}$ ) were placed on the circumference of an imaginary circle (radius = $1.0 \mathrm{deg}$ ) around a fixation point. The five elements were equally spaced, and one of the elements was at the 12 o'clock position. Each of the eight colors was used six times as the target, once on each of the five positions and an additional time on one of the five positions. The latter position was chosen randomly with the constraint that, over all targets, the five positions were used approximately equally of ten. The other member of the pair from which the target stemmed was never presented simultaneously with the target. From one of the three remaining pairs, no member was in the display. Both members of the two remaining pairs were on the display, one member adjacent to the target and one member one position removed from the target. Over the 48 displays, the positions of the four irrelevant items were balanced as far as possible. Figure 1 shows an example of a stimulus card. The first number in the code $x, y$ stands for the pair, and the second number for the item in the pair. Color 3,2 is the target; Color 3,1 and Pair 1 (i.e., 1,1 and 1,2) are not on the display.

For each of the 48 displays, a separate corresponding barmarker card was made. The black barmarker started at the fixation cross, and, when shown in the tachistoscope, pointed at the position of the target. The length of the barmarker was $.5 \mathrm{deg}$ of visual angle.

\section{Analysis}

The stimuli described above allow a descriptive classification of responses in four or five categories. We start, for explanatory purposes, with the classification in four categories. The categories are: (1) correct responses (i.e., responding with the name of the color indicated by the barmarker); (2) visual confusions

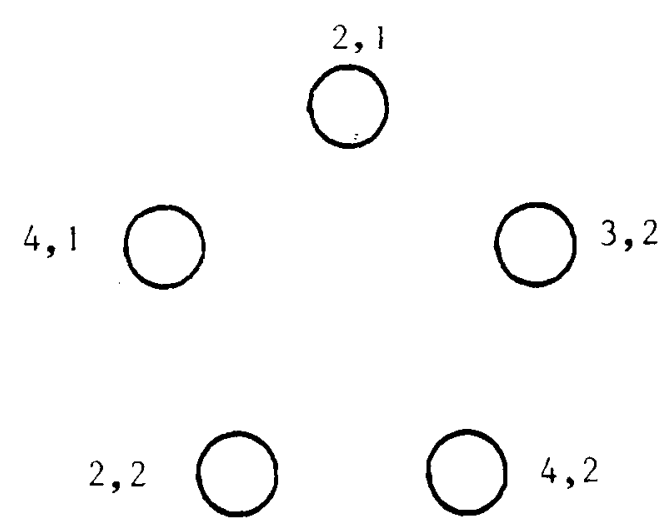

Figure 1. Example of a stimulus card. The first number of the code stands for one of the four pairs of colors, and the second number for the member of a pair ( 1 or 2 ). Color 3,2 is the target. Color 3,1 and Pair 1 (i.e., 1,1 and 1,2) are not on the stimulus. (i.e., responding with the name of the other color of the pair from which the target stemmed); (3) intrusions (i.e., responding with the name of one of the two colors of the pair not on the stimulus card); and (4) location errors (i.e., responding with the name of a color on the stimulus card but not indicated by the barmarker).

As far as causes of responses are concerned, we also distinguish four categories: (1) correct responses (resulting from correct identification of the color indicated by the barmarker), (2) confusions (resulting from genuine misidentification of the color indicated), (3) pure guesses (resulting from random guessing or from misidentifications approaching or equaling random guessing), and (4) location errors (resulting from identification or genuine misidentification of an item in the display other than the item indicated by the barmarker).

In Table 1, the decomposition of the observations in the four descriptive categories (the row headings) according to their inferred causes (the column headings) is presented. In fact, Table 1 contains four equations with four unknowns (A, B, Y, and $n$ ). With regard to causes, the total number of observations is partitioned into three groups: (1) correct identifications of the target (A), (2) genuine misidentifications of the target (B), and the rest of the observations, consisting of pure guesses, misidentifications equaling pure guesses, and, possibly, location errors (Y). Of course, with this partitioning, correct identifications (A) contribute only to the descriptive category correct responses. Because of the items and displays used in the present experiments (see the description in the previous paragraphs), it is reasonable to assume that genuine misidentifications of the target (B) contribute only to the descriptive category confusions. The distribution of pure guesses plus location errors $(\mathrm{Y})$ over the four descriptive categories, and especially the function of the parameter $\mathbf{n}$ in the ratios, requires further explanation. It is possible to distinguish three cases.

First, assume that there are no location errors and that $Y$ is an estimate of pure guesses only. Then it is reasonable to assume that these pure guesses are distributed randomly over the alternatives in the four descriptive response categories. Because there are, respectively $1,1,2$, and 4 responses in these categories, the pure guesses $(\mathrm{Y})$ will be distributed in the proportion $1 / 8,1 / 8,2 / 8$, and $4 / 8$. In other words, if there are only pure guesses that are distributed randomly over the four response categories, then $n=0$, or no parameter $n$ is needed for solving the equations presented in Table 1 .

Second, assume that there are, besides pure guesses, also a number of genuine location errors. Because of the items and the stimulus displays we used (see previous paragraphs), it is reasonable to assume that these errors contribute only to the descriptive category location errors. Now the amount of $\mathrm{Y}$ responses will be distributed over the four response categories in some proportion $1 / 8,1 / 8,2 / 8$, and $X>4 / 8$. Of course, the sum of these probabilities has to be 1 . The parameter $n$ allows for this possibility. With negative values of $n$, more of the $\mathrm{Y}$ responses are allotted to the descriptive category location errors, whereas the ratio of the contributions to the first three categories remains the same. In other words, if more location error responses are obtained than have to be expected on the basis of pure guessing alone, then a value of $n<0$ is needed for solving the equations.

The size of the value of $n$ is a measure of the extra number of location errors expressed in number of response alternatives used for pure guessing. For instance, a value of $n$ of -1 gives the series of proportions $1 / 9,1 / 9,2 / 9$, and $5 / 9$. So, the amount of extra location errors equals the extra amount of location errors that have to be expected on the basis of pure guesses only, if the number of response alternatives is increased from eight to nine, with five of the alternatives resulting in a location error.

There is a third, interesting possibility. Assume that, on some or all trials, subjects have available some or all names, together with correct location information, of the nonindicated items in the display. Assume furthermore that, if subjects have to guess the name of the item indicated by the barmarker, they are 
Table 1

Decomposition of the Number of Observations in the Four Descriptive Response Categories According to Their Causes

\begin{tabular}{llccc}
\hline & & & Inferred Causes \\
\cline { 2 - 4 } \multicolumn{1}{c}{$\begin{array}{c}\text { Descriptive } \\
\text { Categories }\end{array}$} & & $\begin{array}{c}\text { Correct } \\
\text { Identifications }\end{array}$ & $\begin{array}{c}\text { Genuine } \\
\text { Misidentifications }\end{array}$ & $\begin{array}{c}\text { Pure Guesses and } \\
\text { Location Errors }\end{array}$ \\
\hline $\begin{array}{l}\text { Correct Responses } \\
\text { Confusions } \\
\text { Intrusions }\end{array}$ & $=$ & $\mathrm{A}$ & $+[1 /(8-\mathrm{n})] \mathrm{Y}$ \\
Location Errors & $=$ & & $\mathrm{B}$ & $+[1 /(8-\mathrm{n})] \mathrm{Y}$ \\
{$[2 /(8-\mathrm{n})] \mathrm{Y}$} \\
{$[(4-\mathrm{n}) /(8-\mathrm{n})] \mathrm{Y}$}
\end{tabular}

Note-See text for further explanation.

reluctant to respond with one of these incorrect names. (Because there were no repeats in our displays, this is an optimal strategy.) Then, the set of alternatives for pure guessing is reduced. The $Y$ responses will be distributed over the four descriptive response categories in some proportion $1 / 8,1 / 8,2 / 8$, and $X<4 / 8$. Again, the parameter $n$ allows for this possibility. With positive values of $n$, fewer of the $\mathrm{Y}$ responses will be allotted to the descriptive category location errors. In other words, if subjects make fewer location errors than are to be expected with pure guessing only, or if subjects are reluctant to respond with the name of an item on the display not indicated by the barmarker, then a value of $\mathrm{n}>\mathbf{0}$ is needed for solving the equations. In this case, the value of $\mathrm{n}$ is a direct estimate of the number of responses resulting in location errors, excluded from the set of eight alternatives available for pure guessing. For instance, a value of $\mathbf{n}$ of 1 gives the series of proportions $1 / 7,1 / 7,2 / 7$, and $3 / 7$, indicating that subjects behave as if only seven, instead of eight, alternatives are available for guessing, with three of the alternatives resulting in a location error.

Fortunately, the four equations in four unknowns (A, B, $\mathrm{Y}$, and $\mathrm{n}$ ), presented in Table 1 , can be easily solved. So we can obtain the relevant information with regard to the underlying causes of responses. It is possible, however, to separate further the descriptive category location errors into two categories: near location errors (responding with the name of a color adjacent to the target) and far location errors (responding with the name of a color one position removed from the target). Making this distinction has the advantage that we can estimate values of $\boldsymbol{n}$ for near location errors $\left(n_{1}\right)$ and far location errors $\left(n_{2}\right)$ separately. We only have to replace location errors $=$ $[(4-n) /(8-n)] Y$ with near location errors $=\left[\left(2-n_{1}\right) i\right.$ $\left.\left(8-n_{1}-n_{2}\right)\right] Y$ and far location errors $=\left[\left(2-n_{2}\right) /\left(8-n_{1}-\right.\right.$ $\left.\mathrm{n}_{2}\right)$ ] $Y$. The resulting five equations in five unknowns $(A, B, Y$ $n_{1}$, and $n_{2}$ ) can be solved easily (and it is not difficult to see that $n_{1}+n_{2}=n$ )

\section{EXPERIMENT 1}

In this experiment, we investigated whether subjects were able to perform according to the optimal strategy as described under General Method. So, what we wanted to know was whether, if subjects were properly informed about the composition of the stimuli and were instructed not to respond with the name of a color in a wrong position, the number of location errors would be below chance level with probe delay 0 and would move in the direction of chance level with increasing probe delays.

The relevant literature suggested that we would not be successful in obtaining such a result. It has often been reported that the number of location errors is at, or above, chance level with probe delay 0 and further increases to far above chance level with increasing probe delays (see, e.g., Mewhort et al., 1981, Mewhort, Marchetti, Gurnsey, \& Campbell, 1984, and Townsend, 1973). Furthermore, Mewhort et al. (1984) also instructed their subjects not to respond with an item from an incorrect location but to guess a letter that looked as much like the correct one, and nevertheless found an above-chance number of location errors in the relevant subset of data of their experiment.

There is, however, reason for the suspicion that this high absolute number of location errors has not so much to do with the bar-probe task as such, but is mainly caused by the type of stimulus arrays generally used in these tasks (and, of course, by the number of items presented simultaneously, i.e., the number of possible probe positions).

Mewhort et al. (1981), for instance, used eightitem linear arrays. Townsend (1973), however, had already shown that, with eight-item linear arrays, even at probe delay 0 , subjects' ability to correctly locate the probe in space is severely limited. When subjects had only to report the position that the probe had demarcated, more than 25\% (location) errors were made. (See also Mewhort et al., 1981, on channel capacity for linear resolution.) So, the use of such arrays seems to artificially introduce a high intercept value of the function relating location errors to probe delays. It is clear that, under such conditions, an optimal strategy can never show up.

In Experiment 1, we used five-item circular arrays (see General Method). C. W. Eriksen and Rohrbaugh (1970) also used circular arrays and throughout their experiment periodically checked whether subjects could correctly name the clock position at which the barmarker pointed. It appeared that subjects were always correct in reporting that position. So it seems that, with the use of circular arrays, the introduction of an artificially high intercept can be avoided.

Therefore, given our stimulus arrays, it is worthwhile to investigate whether subjects can perform according to the optimal strategy.

\section{Method}

Apparatus. A Scientific Prototype three-channel tachistoscope. Model 320GB, was used to present the stimuli. The subjects initiated stimulus exposure themselves by means of a remote-control switch. The pre- and pustexposure field con- 
sisted of a blank card with a fixation cross in the center. With interstimulus intervals (ISIs) $>0$, this ficld also appeared in the stimulus-probe interval. The stimulus cards, the barmarker cards, and the blank card appeared at a luminance of about $50 \mathrm{~cd} / \mathrm{m}^{2}$.

Materials. Besides the 48 five-item displays and 48 barmarker cards (see General Method), 40 one-element displays ( 8 colors $x$ 5 positions) were prepared. The barmarkers were drawn with India ink on white cards. The colored disks were drawn with colored pencils on white cards. (We were not successful in finding commercially available colored self-adhesive labels or set of colored pens that met the requirements described under General Method.)

Subjects. Eight students of the University of Leiden served as paid subjects. They all had normal or corrected-to-normal vision, and none of them was deficient in color vision. None of them had served in a similar experiment before.

Design. In the main experiment, the stimulus and the barmarker were exposed for $50 \mathrm{msec}$. Four stimulus-probe intervals (i.e., ISIs) were used: 0, 100, 200, and $300 \mathrm{msec}$. Each subject was tested 48 times at each probe delay (i.e., once with the complete set of cards). The order of probe delays over subjects was balanced according to two independent $4 \times 4$ Latin squares. For each probe delay and each subject, the set of 48 stimulus-barmarker combinations was independently randomized.

Procedure. Each subject served on 2 consecutive days. The 1 st day was used for practice only, and the 2 nd day for running the main experiment.

The first part of the practice session was used for familiarizing the subjects with the colors and color names used. First, they were shown the one-element displays outside the tachistoscope and were told the names. Then the one-item displays were shown in the tachistoscope for exposure durations ranging from 300 to $10 \mathrm{msec}$. The subjects had to name the colors. They were corrected when they responded with a wrong name until virtually no between-pair errors were made. The second part of the practice session was used to familiarize the subjects with the stimuli and procedure used in the main experiment. First, the five-element displays were shown outside the tachistoscope. The subjects were explicitly instructed about the rules followed in the construction of the cards. Then the five-element displays were shown for exposure durations ranging from 200 to $50 \mathrm{msec}$ in the tachistoscope. The barmarker followed at intervals ranging between 0 and $500 \mathrm{msec}$. The subjects had to name the color indicated by the barmarker. They were informed about the optimal strategy and were required to never respond with the name of a color in a wrong position. They were corrected when they made an error. In the last part of this practice session, the subjects were trained with the exposure conditions used in the main experiment. In this phase of practice, no further feedback was given.

The 2 nd day was used for the main experiment. At the start of the session, the subjects were reminded of the optimal strategy and were urged not to respond with the name of a color in a wrong position. Before each series of 48 stimuli, five practice trials were presented to familiarize the subjects with the probe delay used. A series lasted about $10 \mathrm{~min}$. Between series, there was a rest period of about $5 \mathrm{~min}$ in which the experimenters arranged the series of 48 stimulus-barmarker combinations in a new random order.

\section{Results}

Identification and error data. The responses were scored as correct or incorrect. The incorrect responses were classified as item errors or as location errors. Item errors are the responses of the name of a color not shown on the trial. Two kinds of item errors are distinguished: confusions and intrusions. A confusion occurs when subjects name the other member of the color pair from which the color indicated by the probe stems. An intrusion is a response of one of the two colors of the pair not shown on the trial.

A location error is a response of a color shown on the trial other than the color indicated by the probe. Two kinds of location errors are distinguished, near location errors and far location errors. A near location error is a response of one of the two items next to the color indicated by the probe. A far location error is a response of one of the two other nonindicated items on the stimulus card.

Table 2 gives, per barmarker interval, the absolute number and, in parentheses, the proportion of responses in each of these classes.

Subsequent analyses of variance showed that for none of the five response categories distinguished in Table 2 was the effect of probe delay significant. The highest $F$ values were obtained for correct responses $[F(3,21)=2.26, p<.12]$ and for confusions $[F(3,21)=$ $1.75, \mathrm{p}<.19]$.

The mean proportion of confusions (.163) and the mean proportion of intrusions (.065) differed significantly $[F(1,7)=58.19, \mathrm{p}<.001]$. The mean proportion of intrusions and the mean proportion of near location errors $(.050)$ did not differ significantly $[F(1,7)=$ $2.15, \mathrm{p}<.20]$, but the mean proportion of intrusions and the mean proportion of far location errors (.030) did $[F(1,7)=28.51, p<.01]$. Furthermore, the mean proportion of near location errors was significantly greater than the mean proportion of far location errors $[F(1,7)=7.72, p<.05]$. In none of the above compari-

Table 2

Absolute Number and, in Parentheses, Proportion of Correct and of Error Reports per Probe Delay

\begin{tabular}{lcccc}
\hline & \multicolumn{4}{c}{ Probe Delay (in Milliseconds) } \\
\cline { 2 - 4 } Response Class & 0 & 100 & 200 & $261(.680)$ \\
\hline $\begin{array}{l}\text { Correct } \\
\text { Error }\end{array} \quad 287(.747)$ & $263(.685)$ & & \\
$\quad$ Item & & & $69(.180)$ & $251(.654)$ \\
$\quad \begin{array}{l}\text { Confusion } \\
\quad \text { Intrusion }\end{array}$ & $54(.141)$ & $66(.172)$ & $25(.065)$ & $61(.159)$ \\
$\quad$ Location & $18(.047)$ & $29(.076)$ & & $28(.073)$ \\
$\quad$ Near & $14(.036)$ & $19(.050)$ & $16(.042)$ & $28(.073)$ \\
$\quad$ Far & $11(.029)$ & $7(.018)$ & $13(.034)$ & $16(.042)$ \\
\hline
\end{tabular}

Note-See text for further explanation (Experiment 1 ). 
sons did the effect of probe delay and the interactions involving probe delay appear significant.

Identification and error analysis. Because of the very small absolute number of intrusions, near location errors, and far location errors (see the last three rows in Table 2), the scatter in the same raw data as a result of chance fluctuations, and the high sensitivity of our method of analysis for such fluctuations, the analysis of the data in Table 2 appeared not to be very enlightening. In order to increase the number of observations and to diminish the effect of chance fluctuations for the present experiment, we first calculated "running sums" for adjacent probe delays. Table 3 presents these sums in the same format that was used in Table 2.

In Table 4 , the results of the analysis elaborated under General Method are summarized. The six rows in this table present, respectively, (1) the number (and, in parentheses, the proportion) of correct reports, corrected for guessing (i.e., A), (2) the number and proportion of confusions corrected for guessing (i.e., B), (3) the rest of the reports (Y), consisting of incorrect, nonconfusion reports, (4) the parameter $n_{1}$, measuring the deviation from pure guessing for the two items on the locations next to the target, (5) the parameter $n_{2}$, measuring the deviation from pure guessing for the other two items on the stimulus, and (6) the sum of

Table 3

A.bsolute Number of Correct and of Error Reports Summed Over Adjacent Probe Delays (i.e., "running sums"), and, in Parentheses, the Proportions of These Reports

\begin{tabular}{lrrr}
\hline & \multicolumn{3}{c}{ Probe Delay (in Milliseconds) } \\
\cline { 2 - 4 } Response Class & $0+100$ & $100+200$ & $200+300$ \\
\hline $\begin{array}{l}\text { Correct } \\
\text { Error } \\
\quad \text { Item }\end{array}$ & $550(.716)$ & $524(.682)$ & $512(.667)$ \\
$\quad$ Confusion & $120(.156)$ & $135(.176)$ & $130(.169)$ \\
$\quad$ Intrusion & $47(.061)$ & $54(.070)$ & $53(.069)$ \\
$\quad$ Location & & & \\
$\quad$ Near & $33(.043)$ & $35(.046)$ & $44(.057)$ \\
$\quad$ Far & $18(.023)$ & $20(.026)$ & $29(.038)$ \\
\hline
\end{tabular}

Note-See text for further explanation (Experiment 1).

Table 4

Estimated Number of Correct Reports (A), Confusions (B), Incorrect Nonconfusion Reports (Y) (and, in Parentheses, the Proportions of These Reports), and the Estimated Guessing Parameters, $n_{1}, n_{2}$, and Their Sum, $n_{1}+n_{2}$

Probe Delay (in Milliseconds)

\begin{tabular}{lccc} 
& $0+100$ & $100+200$ & $200+300$ \\
\hline Correct Reports (A) & $526.5(.686)$ & $497.0(.647)$ & $485.5(.632)$ \\
Confusions (B) & $96.5(.126)$ & $108.0(.141)$ & $103.5(.135)$ \\
$\mathrm{Y}$ & $145.0(.189)$ & $163.0(.212)$ & $179.0(.233)$ \\
$\mathrm{n}_{1}$ & .596 & .704 & .340 \\
$\mathrm{n}_{2}$ & 1.234 & 1.259 & .906 \\
$\mathrm{n}_{1}+\mathrm{n}_{2}=\mathrm{n}$ & 1.830 & 1.963 & 1.246 \\
\hline
\end{tabular}

Note-See text for further explanation (Experiment 1). $n_{1}$ and $n_{2}$, that is, $n$, measuring the overall deviation from pure guessing for items presented simultaneously with the target.

\section{Discussion}

The prime purpose of the present experiment was to investigate whether subjects can perform according to the optimal strategy if they are appropriately informed and instructed. The fact that the number of intrusions is somewhat larger than the number of near location crrors and significantly exceeds the number of far location errors shows that subjects indeed can. The values of $\mathbf{n}$ in Table 4 substantiate this conclusion. As indicated under General Method, positive values of $n$ indicate a bias against guessing the name of elements on nonindicated positions in the array. The rather large values of $n$ indicate that appreciably fewer location errors are made than have to be expected on the basis of chance alone. The figures in Table 4 also suggest that the value of $n$ decreases with increasing probe delay. This trend indicates that the number of location errors increases faster than the number of intrusions with increasing probe delays, as has been reported more often in the literature (cf. e.g., C. W. Eriksen \& Rohrbaugh, 1970; Mewhort et al., 1981). Nevertheless, even with probe delay $200+300$, the values of $\mathbf{n}$ are still greater than 0 , indicating below-chance use of the names of nonindicated colors for guessing. In this way, the present experiment shows that, if appropriate (circular, five-item) arrays are used and if subjects are properly informed and instructed, they can perform according to the optimal strategy.

Three further features of the data are worthy of note; the absence of significant probe-delay effects for the descriptive response categories, the number of confusions, and the difference in the number of near and far location errors.

The probe-delay effect was not significant in any of the descriptive response categories. This result is somewhat surprising because, especially for correct responses, strong probe-delay effects are reported in the literature. One further observation is relevant. After the experiment, the subjects reported that it was extremely difficult to obey the instructed strategy, especially with the shortest probe delays. Some stated that they changed strategy during the test series. It is likely that such variability in strategies introduces an appreciable amount of within- and between-subjects variance and that the increased variance-together with the relatively low number of observations and high average level of accuracyprevented the probe-delay effects from appearing sig. nificant.

Tables $2-4$ and the analyses of variance show that an appreciable number of what we classify as confusions occur. This feature of the data strongly suggests that we were successful in channeling the main number of visual confusions to the other member of the pair from which the element indicated by the barmarker stemmed. The 
function relating confusions to probe delays is negatively accelerated and even ultimately seems to decrease, a point to which I shall return further on.

The tables and the analyses of variance show that the number of near location errors exceeds the number of far location errors. Such a result has been reported previously in the literature. In the earlier experiments, however, either a larger number of elements was presented simultaneously (Snyder, 1972, used 12 elements; C. W. Eriksen \& Rohrbaugh, 1970, used 4, 8, and 12 elements but did not report on location errors for the 4-element display; Dick, 1974, used 6 elements; and Mewhort et al., 1981, used 8 elements), or linear arrays instead of circular arrays were used (Dick, 1974; Mewhort et al., 1981). A significant difference between near and far location errors for a 5 -element circular array is therefore rather surprising.

Under General Discussion, we return to the more general implications of the present results for the three theories outlined in the introduction.

\section{EXPERIMENT 2}

The prime purpose of Experiment 2 was to investigate whether subjects also perform according to the optimal strategy if appropriate (circular, five-element) arrays are used, but when those subjects are neither explicitly informed about the composition of the arrays, nor urged to perform according to such a strategy. Experiment 1 showed that subjects can perform according to the optimal strategy when instructed to do so. Of course, for theoretical purposes, it is essential to know whether subjects also spontaneously adopt this strategy.

A further purpose of Experiment 2 was to establish more firmly the probe delay effects suggested by the results of Experiment 1. Of special interest, here, is the number of what we called "confusions" as a function of probe delay. Experiment 1 suggests that the number first increases and then decreases. This result was not expected, but, nevertheless, this time course suggests that the confusions category really provides an estimate of the number of genuine visual confusions. Assume that performance in partial-report bar-probe tasks depends for one reason or another on the quality of the visual representation at the moment that the item to be reported is selected, as suggested by the first two models described in the introduction. Then, if the quality of the visual representation progressively deteriorates over time, ultimately a condition at which visual confusions approach or equal pure guesses will be reached. Before that stage is reached, however, visual confusions will contribute mainly to our confusions category, and an increase in the number of these confusions will be observed with some increase in probe delay. With still longer probe delays, visual confusions will no longer contribute mainly to that category. but will be distributed as random guesses over the different response categories. So, after an increase, ultimately a decrease in the number of observed confusions is to be expected. (See Mewhort et al., 1984, for a similar argument.)

To establish the various probe-delay effects more firmly, especially the probe-delay effect for the category confusions, we increased (quadrupled) the number of observations per subject.

\section{Method}

Material. The apparatus and stimuli were the same as those in Experiment 1.

Subjects. Eight students (not the same as those in Experiment 1) of the University of Leiden served as paid subjects. All had normal or corrected-to-normal vision, and none was deficient in color vision. None had served in a similar experiment before, and none knew about the type of experiments we were performing.

Design. In the experiment, the exposure conditions were the same as those in Experiment 1. Each subject served on 4 consecutive days. Each day, a subject was tested 48 times at each probe delay (i.e., once with the complete set of cards). The order of probe delays per subject per day, over days, and over subjects was balanced by using $4 \times 4$ Latin squares. For each subject, day, and probe delay, the set of 48 stimulus-barmarker combinations was randomized independently.

Procedure. Each subject served for 5 days. The 1 st day was for practice only. Practice was the same as in Experiment 1, except that nothing was indicated about the rules for the construction of the arrays or about an optimal strategy. The subjects were instructed simply to respond with the name of the color indicated by the barmarker. The same instructions were given at the start of each session in the main experiment. In all other regards, the experiment was similar to Experiment 1.

\section{Results}

Identification and error data. The responses were scored in the same way as in Experiment 1. Table 5 gives, per barmarker interval, the absolute number and, in parentheses, the proportion of responses in each of the classes distinguished.

Subsequent analyses of variance showed significant probe-delay effects for correct responses $[F(3,21)=$ $14.51, \mathrm{p}<.001]$, for confusions $[\mathrm{F}(3,21)=10.62$, $\mathrm{p}<.001]$, and for near location errors $[\mathrm{F}(3,21)=7.85$, $\mathrm{p}<.01]$. For near plus far location errors, the probedelay effect was significant $[\mathrm{F}(3,21)=6.58, \mathrm{p}<.01]$. For intrusions, the probe-delay effect approached significance $[F(3,21)=2.74, p<.07]$, and for far location errors, the probe-delay effect was not significant $[\mathrm{F}(3,21)$ $=2.30]$. A two-tailed t test showed that the decrease in the mean proportion of confusions from probedelay 200 to probe-delay 300 was significant $[\mathrm{t}(7)=$ $2.38, \mathrm{p}<.05]$.

The mean proportion of confusions (.161) and the mean proportion of intrusions (.075) differed significantly $[F(1,7)=102.81, p<.001]$. The mean proportion of intrusions and the mean proportion of near location errors (.036) and the mean proportion of intrusions and the mean proportion of far location errors (.032) just differed significantly $[F(1,7)=5.70, p<.05$, and $F(1,7)=11.09, p<.05$, respectively $]$. The differ- 
Table 5

Absolute Number and, in Parentheses, Proportion of Correct and of Error Reports per Probe Delay

\begin{tabular}{lcccc} 
& & & & Probe Delay (in Milliseconds) \\
\cline { 2 - 5 } Response Class & 0 & 100 & 200 & 300 \\
\hline $\begin{array}{l}\text { Correct } \\
\text { Error }\end{array} \quad$ Item & $1160(.755)$ & $1109(.722)$ & $1016(.661)$ & $994(.647)$ \\
$\quad$ Confusion & & & & \\
$\quad$ Intrusion & $208(.135)$ & $240(.156)$ & $287(.187)$ & $256(.167)$ \\
$\quad$ Location & $100(.065)$ & $98(.064)$ & $121(.079)$ & $139(.090)$ \\
$\quad$ Near & $34(.022)$ & $46(.030)$ & $57(.037)$ & $85(.055)$ \\
$\quad$ Far & $34(.022)$ & $43(.028)$ & $55(.036)$ & $62(.040)$ \\
\hline
\end{tabular}

Note-See text for further explanation (Experiment 2).

ence between the mean proportion of near location errors and the mean proportion of far location errors was far from significant $[F(1,7)=0.60]$. In accordance with the probe-delay effects described in the previous paragraph, in all these comparisons the effect of probe delay was highly significant. From the interactions involving probe delay, only the interaction for the comparison between proportions of confusions and proportions of intrusions approached significance $[F(3,21)=$ $3.06, p<.10]$. In this interaction, the nonlinear relation between proportion of confusions and probe delay is involved.

Identification and error analysis. Table 6 gives the results of the analysis outlined under General Method in the same format as in Experiment 1.

In Table 7, the results for only the 1 st day of practice are presented.

\section{Discussion}

The prime purpose of Experiment 2 was to investigate whether subjects also spontaneously adopt the optimal strategy. The observation that the number of intrusions significantly exceeds the number of near and the number of far location errors for all probe delays shows that they indeed do (see Table 5). The same result is reflected in the positive values of $n$ in Table 6 . Again, the rather large values of $n$ indicate that appreciably fewer location errors were made than have to be expected on the basis of chance alone. Table 6 furthermore clearly shows that the values of $n$ decrease with increasing probe delays. So, it appears that with the type of stimulus arrays we used, the number of location errors starts at a level far below chance and moves in the direction of chance (i.e., $\mathrm{n}=0$ ) with increasing probe delays. Table 7 shows that essentially the same pattern of results was already apparent on the 1 st day of practice. So, we can conclude that subjects do not arrive gradually at the optimal strategy, but start using this strategy at the very beginning of this partial-report bar-probe task.

A further purpose of Experiment 2 was to establish more firmly the function relating number of confusions to probe delays. Table 5 and Table 6 clearly show, and this result was confirmed by the statistical analysis, that this relation is indeed nonmonotonic. The number of confusions first increases and then decreases. To the theoretical importance of this observation we briefly return under General Discussion. (Table 7 shows that, in Experiment 2, this nonmonotonic relation is not yet apparent on the 1st day of practice. However, these data show that the function is negatively accelerated; corrected for guessing, the number of confusions equals $40,51,57.5$, and 59.5 for probe delays 0 to 300 , respectively. The small absolute number of confusions per day make it impossible to say something sensible about this slight deviation of the results obtained on the 1st day of practice from the overall pattern of results obtained.)

Two further aspects of the data are worth brief mention. First, the analyses of variance showed signifi-

Table 6

Estimated Number of Correct Reports (A), Confusions (B), Incorrect Nonconfusion Reports (Y) (and, in Parentheses, the Proportions of These Reports), and the Estimated Guessing Parameters, $n_{1}, n_{2}$, and Their Sum, $n_{1}+n_{2}$

\begin{tabular}{lcccc}
\hline & \multicolumn{3}{c}{ Probe Delay (in Milliseconds) } \\
\cline { 2 - 4 } & 0 & 100 & 200 & 300 \\
\hline Correct Reports (A) & $1110.0(.723)$ & $1060.0(.690)$ & $955.5(.622)$ & $924.5(.602)$ \\
Confusions (B) & $158.0(.103)$ & $191.0(.124)$ & $226.5(.147)$ & $186.5(.121)$ \\
$\mathrm{Y}$ & $268.0(.174)$ & $285.0(.186)$ & $354.0(.230)$ & $425.0(.277)$ \\
$\mathrm{n}_{1}$ & 1.320 & 1.061 & 1.058 & .777 \\
$\mathrm{n}_{2}$ & 1.320 & 1.122 & 1.091 & 1.108 \\
$\mathrm{n}_{1}+\mathrm{n}_{2}=\mathrm{n}$ & 2.640 & 2.183 & 2.149 & 1.885 \\
\hline
\end{tabular}

Note-See text for further explanation (Experiment 2). 
Table 7

Absolute Number and, in Parentheses, Proportion of Correct and of Error Reports per Probe Delay

\begin{tabular}{lcccc}
\hline & \multicolumn{4}{c}{ Probe Delay (in Milliseconds) } \\
\cline { 2 - 5 } Response Class & 0 & 100 & 200 & 300 \\
\hline Correct & $277(.721)$ & $266(.693)$ & $242(.630)$ & $231(.602)$ \\
Error & & & & \\
$\quad$ Item & & & & \\
$\quad$ Confusion & $55(.143)$ & $65(.169)$ & $34(.193)$ & $77(.201)$ \\
$\quad$ Intrusion & $30(.078)$ & $28(.073)$ & & $35(.091)$ \\
$\quad$ Location & $13(.034)$ & $13(.034)$ & $18(.047)$ & $22(.057)$ \\
$\quad$ Near & $9(.023)$ & $12(.031)$ & $17(.044)$ & $19(.049)$ \\
$\quad$ Far & & & & \\
\hline
\end{tabular}

Note-See text for further explanation (Experiment 2, Day 1).

cant probe-delay effects for the descriptive categories correct responses and location errors, and an effect approaching significance for intrusions. The size and direction of these effects are consistent with the results generally reported with this type of task (cf., e.g., Mewhort et al., 1981). So it appears that, given a sufficient number of observations (and, possibly, a stable strategy) and the stimulus arrays and procedure that we used, most of the characteristic features generally obtained with partial-report bar-probe tasks can be produced.

Second, in the present experiment, the difference between the amount of near and far location errors was far from significant, as was the interaction between the two types of location errors and probe delays. On the one hand, this result is not surprising, because fiveelement circular arrays allow high spatial accuracy. C. W. Eriksen and Rohrbaugh (1970) reported a difference between near and far location errors for eightelement arrays but not for four-element arrays. On the other hand, the significant difference between near and far location errors with the same arrays in Experiment 1 makes this result rather surprising. Table 7 shows that, in Experiment 2, on the 1st day of practice the difference between the two types of location errors is already very small. So, different amounts of practice cannot account for the different results obtained in the two experiments. In one way or another, the different instructions and the resulting differences in strategies have to account for this difference in the results.

\section{GENERAL DISCUSSION}

In this section we discuss the implications of the results of the experiments reported, for the three theories described in the introduction. First, we discuss the preversus postcategorical selection issue; then we discuss the role of the precategorical store (A) in this type of task. We end this section with a further discussion of location errors and of postcategorical filtering.

As stated in the introduction, Mewhort et al.'s (1981) and Van der Heijden's (1981) models are postcategorical selection models. In both models, identified information is selected for report. These two models have to predict that effects of irrelevant identified information can show up in the data and will be found if the data are properly analyzed. In the orthodox, precategorical selection models, it is assumed that selection is from among unidentified, precategorical information, and therefore no effects of identified irrelevant information are predicted (I return to this issue later).

What our ultimate purpose was in investigating whether subjects can perform according to, and whether they spontaneously adopt, the optimal strategy should now be clear. Performing according to the optimal strategy-that is, not responding with the name of an item in a wrong position if, for one reason or another, the correct name is not available-is possible only if both identity and location information for the items in the wrong positions are available. The observation that subjects can perform according to the optimal strategy (Experiment 1) and spontaneously adopt the optimal strategy (Experiment 2) indicates that this information is indeed available. Therefore, we have to conclude that selection is postcategorical selection, that is, selection from among identified information.

There is a way for adherents of precategorical selection models to try to escape from this conclusion. The concept "nonselective transfer" has then to be introduced within the precategorical selection view. When the probe is delayed, there is a period of time during which information in store $\mathrm{A}$ is decaying and during which the subject does not know which item he or she will be required to report. During this period, the subject transfers items from store $A$ to store $B$ without reference to the cue, that is, engages in what is called nonselective transfer. (See, e.g., Averbach \& Coriell, 1961; for a discussion, see Coltheart, 1977.) In this way, precategorical selection models can also account for our observation that subjects have available and therefore can use more information than the information requested by the probe. So, in principle, a precategorical selection model also can account for the observation that subjects perform according to the optimal strategy.

For three reasons, this explanation will not do for the present experiments, however. (Remember that 
positive values of $\mathbf{n}$ directly estimate the number of responses resulting in location errors, excluded from the set of eight alternatives available for pure guessing; i.e., positive values of $n$ directly estimate the number of irrelevant elements that subjects have identified and rejected as response alternatives if they have to guess the identity of the element indicated by the barmarker.) First, it appears that at probe delay 0 , subjects have available at least 2.64 of the 4 irrelevant items (the value of $n$ in Table 6 ). It seems that this value at this probe delay is much too high for nonselective transfer, especially because colors, not letters or digits, were used as the stimulus items. Second, the value of $n$ decreases with increasing probe delays (from 2.64 to 1.89 in Table 6), whereas with nonselective transfer, $\mathrm{n}$ should increase with increasing probe delay: the longer the stimulus-probe interval, the more time for nonselective transfer. Third, the proportions available of the four nonindicated elements in the array (estimated with $\mathrm{n} / 4$ in Table 6) equal $.66, .55, .54$, and .47 for the four probe delays, respectively. The corresponding proportions of correct reports equal $.72, .69, .62$, and .60 (see Table 6). So, the differences are rather small, indicating only a small advantage for the relevant information. Furthermore, irrelevant information seems to disappear somewhat faster than relevant information. To make these features more apparent, we fitted exponential decay functions of the form $\mathrm{Pe}^{-\alpha t^{\prime}}$ to the proportions for the indicated and the nonindicated items separately. Because Averbach and Coriell (1961) and C. W. Eriksen and Collins (1969) estimated probe-processing time to be on the order of $100-200 \mathrm{msec}$, we used stimulus duration + probe delay $+150 \mathrm{msec}$ as the value for $\mathrm{t}^{\prime}$ (i.e., $t^{\prime}$ equals $200,300,400$, and $500 \mathrm{msec}$ ). For the indicated items, the decay function equals $.82 \mathrm{e}^{-.0061}$ $(R=.98)$, and for the nonindicated items, this function equals $.79 \mathrm{e}^{-.001 t^{\prime}}(\mathrm{R}=.96)$. These functions strongly suggest that initially relevant and irrelevant items are processed equally (proportions equal .82 and .79 at $\mathrm{t}^{\prime}=$ 0 , respectively) and that the only difference is in a somewhat slower decay of the relevant information. Both features are consistent with postcategorical selection models (see, e.g., Van der Heijden, 1981, pp. 158-166) and are difficult to handle in terms of precategorical selection models. (A possible escape for adherents of precategorical selection models is to assume that subjects continue nonselective transfer after selective transfer has not been successful. This new, post hoc explanation seems too farfetched to deserve further discussion.)

Taken together, it seems that the two experiments provide strong evidence in favor of postcategorical selection models and show that the orthodox view of iconic memory has to be rejected. In passing, it is interesting to note that the experiments also convincingly show that effects of unwanted irrelevant information can also be demonstrated, if only accuracy is used as the dependent variable. So, effects of unwanted information do not show up only as interference or facilitation in experiments having latency as the main dependent variable. Furthermore, it is interesting to note that the present analysis shows that it is possible to estimate the amount of irrelevant information available directly from the incorrect responses. So, it is not necessary to infer this amount indirectly from the correct responses, as in Sperling's (1960) sample-to-whole procedure. Finally, it is interesting to note that the present analysis provides information about the (parallel) processing of information that is consistent with the evidence about the (parallel) processing of information obtained in visual search tasks (see, e.g., Van der Heijden, La Heij, \& Boer, 1983).

The above analysis leaves us with the two postcategorical selection models described in the introduction. As stated there, these two models differ with regard to the issue of at what level selective attention operates. Van der Heijden's (1981) model states that selective attention works upon information in store A. Therefore, this model has to predict that decay of information in store A will be apparent in the data. In other words, the error pattern will reflect the state of the representation in store $\mathrm{A}$, and therefore, the model predicts, with in creasing probe delay, besides an increase in the number of location errors, an appreciable increase in the number of item errors (i.e., errors consisting of responding with the name of an item not in the array). In Mewhort et al.'s (1981) model, it is postulated that selective attention operates in store B. It is assumed that in store B only spatial uncertainty increases over time and that there is virtually no loss of identity information. Therefore, this model predicts an appreciable increase in the number of location errors with increasing probe delays and virtually no increase in the number of item errors (see Mewhort et al., 1981, pp. 52-53).

The data obtained in the two experiments are very clear with regard to this issue. In Experiment 1, the proportions of item errors (i.e., in our experiments, the sum of the proportions for confusions and intrusions) equal $.188, .248, .245$, and .232 for the four probe intervals. So the maximum increase, reached at probe delay 100 , equals .060 , and the ultimate increase, at probe delay 300 , is .044 . The corresponding proportions for (near plus far) location errors are $.065, .068, .076$, and .115 , that is, a maximum and ultimate increase of .050 at probe delay 300. In Experiment 2, the proportions of item errors equal $.200, .220, .266$, and .257 , showing a maximum increase of .066 at probe delay 200 and an ultimate increase of .057 . For location errors, the proportions are $.044, .058, .073$, and .095 , showing a maximum and ultimate increase of .051 . Furthermore, if we recall that there are three alternatives for item errors and four alternatives for location errors, then these figures make clear that in our experiments the increase in item errors is at least as strong as the increase in location errors. This feature of the data, together with the nonmonotonic relation between confusions and probe delays (as elaborated in the introduction to Experi- 
ment 2 , such a relation stongly suggests that confusions reflect the state of the information in store $\mathrm{A}$ ), provides strong, direct empirical evidence against Mewhort et al.'s (1981) model.

Keele and Chase (1967) also demonstrated that at least a substantial subset of the errors reflects the state of the information in store $\mathrm{A}$ in a partial-report barprobe task with letters and digits as items. They classified all errors into a matrix of presented letter by error response. These matrices were correlated with a matrix of visual similarity between letter pairs. The correlations for short (0-, 50-, and 100-msec) medium (250- and $500-\mathrm{msec})$, and long $(1,000-$ and $5,000-\mathrm{msec})$ cue delays were $.37, .17$, and .17 , respectively. No evidence of acoustic confusions was found. Furthermore, Keele and Chase's observation that there are more visual confusions at short delays than at intermediate delays is completely consistent with our finding that the number of confusions ultimately decreases.

The analysis presented up to now leaves us only with the postcategorical filtering model. Before we comment on this model, one issue has to be discussed briefly.

Whatever the results of our experiments and analyses may be, the fact remains that Mewhort et al. (1981), Mewhort et al. (1984), and Townsend (1973), for example, found a much stronger increase in location errors than in item errors with increasing delays. (This was the major impetus for Mewhort et al., 1981, to develop their postcategorical selection model.) So, we have to look briefly at whether alternative explanations for this pattern of results can be found. Two possible explanations will be described.

First, it cannot be ruled out that, with eight-element linear arrays, relating the position of the probe to the position of the items-an operation performed in store $A$, according to Van der Heijden $(1981$, p. 106)-becomes more and more difficult with increasing probe delays. At first sight, the results of Townsend's (1973) positionnaming task mentioned in the introduction to Experiment 1 seem to argue against such an explanation. In Townsend's experiment, no effect at all of probe delay was found when subjects had only to indicate the position at which the barmarker pointed. For this experiment, however, there is no reason to assume that, for task performance, information from two successive fields had to be integrated, related, or compared in store A. Estimating the absolute position of the barmarker in its own field suffices in this task. Note that this interpretation of the position-naming results also explains why no effect of probe delay was found.

Second, our data hint at another, intriguing explanation of the dissociation between the two types of errors as a function of probe delay. In our experiments, there were 8 response alternatives. From Table 6, it appears that, with probe delay 0 , if subjects had to guess, about 2.6 of the 4 nonindicated colors in the array were excluded from the set of alternatives used for guessing.
So, from the $8,5.4$ alternatives remain. From these $5.4,3$ result in item errors (a proportion of .56) and 1.4 in location errors (.26). The last alternative results in a correct guess (.19). With probe delay $300,1.9$ alternatives are excluded, and 6.1 remain. From these $6.1,3$ again result in item errors (.49), 2.1 result in location errors (.34), and 1 in a correct response (.16). So, in our experiment, the proportion of item errors resulting from guessing decreases from .56 to .49 and the proportion of location errors increases from .26 to .34 with a probe-delay increase from 0 to 300 . With a small increase in the number of guesses with increasing probe delay from 0 to 300 (in the present example, an increase of about $14 \%$ ), guesses will not result in an increase of item errors or in a substantial increase in location errors. It is not clear whether such an explanation also applies if eight-letter linear arrays are used. On the one hand, with such displays there are 18 alternatives for item errors and only between 0 and 7 alternatives for location errors. On the other hand, it is possible that, with letters instead of colors, the initial value of $n$ is higher and, furthermore, that $n$ decreases faster with increasing probe delays. If so, then subjects' decreasing reluctance to guess names of letters occupying the wrong positions can explain a large part of the dissociation upon which Mewhort et al.'s (1981) model is primarily based.

Taken altogether, it seems that the results of the two experiments reported here favor the postcategorical filtering model. The essence of this model is the assumption that precategorical information (i.e., information in store A) is used for postcategorical selection (i.e., selection of information in store B). In combining Broadbent's (1958) concept of selection (i.e., filtering) and Morton's (1969) concept of processing of information (i.e., the logogen system), the model tries to be a minimal reformulation of the orthodox iconic memory view (see, e.g., Coltheart, 1975). The essential change proposed is to replace words like "processing," "identifying," and "categorizing" in the orthodox view with "filtering," "attending," and "selecting." Nevertheless, this small change leads to considerable theoretical problems (see, e.g., Coltheart, 1980). For some of these problems and for possible solutions, the reader is referred to Van der Heijden (1981). The new theoretical problem the present research adds is that of how to model subjects' reluctance to use as a response the names of items in wrong positions, if they have to guess. $^{2}$

Our present research is concerned with the problem of whether, with letters as items, the same results are obtained that are obtained with colors as items. After all, it remains possible that Mewhort et al.'s (1981) "dual-buffer" account is adequate only for letter stimuli and does not apply to color-stimuli. Then, however, the research reported here reveals an important and unexpected difference between letters and colors in partial-report bar-probe tasks. 


\section{REFERENCES}

Averbach, E., \& Coriell, A. S. (1961). Short-term memory in vision. Bell System Technical Journal, 40, 309-328.

Broadbent, D. E. (1958). Perception and communication. London: Pergamon Press.

Coltheart, M. (1975). Iconic memory: A reply to Professor Holding. Memory \& Cognition, 3, 42-48.

Coltheart, M. (1977). Contemporary models of the cognitive processes. I: Information input and storage. In V. Hamilton \& M. D. Vernon (Eds.), The development of cognitive processes. London: Academic Press.

Coltheart, M. (1980). Iconic memory and visible persistence. Perception \& Psychophysics, 27, 183-228.

Dick, A. O. (1974). Iconic memory and its relation to perceptual processing and other memory mechanisms. Perception \& Psychophysics, 16, 575-596.

Di Lollo, V. (1978). On the spatio-temporal interactions of brief visual displays. In R. H. Day \& G. V. Stanley (Eds.), Studies in perception. Perth: University of Western Australia Press.

Eriksen, B. A., \& Eriksen, C. W. (1974). Effects of noise letters upon the identification of a target letter in a nonsearch task. Perception \& Psychophysics, 16, 143-149.

Eriksen, C. W., \& Collins, J. F. (1969). Temporal course of selective attention. Journal of Experimental Psychology, 80, 254-261.

Eriksen, C. W., \& Hofrman, J. E. (1973). The extent of processing of noise elements during selective encoding from visual displays. Perception \& Psychophysics, 14, 217-224.

Eriksen, C. W., \& Rohrbaugh, J. W. (1970). Some factors determining efficiency of selective attention. American Journal of Psychology, 83, 330-343.

GatTI, S. V., \& EGETH, H. E. (1978). Failure of spatial selectivity in vision. Bulletin of the Psychonomic Society, 11, 181-184.

KeELE, S. W., \& Chase, W. G. (1967). Short-term visual storage. Perception \& Psychophysics, 2, 383-386.

McClelland, J. L., \& Rumelhart, D. E. (1981). An interactive activation model of context effects in letter perception: I. An account of basic findings. Psychological Review, 88, 375-407.

Mewhort, D. J. K., Campbell, A. J., Marchetti, F. M., \& CAmpBell, J. I. D. (1981). Identification, localization, and "iconic memory": An evaluation of the bar-probe task. Memory \& Cognition, 9, 50-67.

Mewhort, D. J. K., Marchetti, F. M., Gurnsey, R., \& CampBELL, A. J. (1984). Information persistence: A dual-buffer model for initial visual processing. In H. Bouma \& D. G. Bouwhuis (Eds.), Attention and performance $X$. Hillsdale, $\mathrm{NJ}$ : Erlbaum.

Morton, J. (1969). Interaction of information in word recognition. Psychological Review, 76, 165-178.

SNYDE R, C. R. R. (1972). Selection, inspection, and naming in visual search. Journal of Experimental Psychology, 92, 428-431.

Sperling, G. (1960). The information available in brief visual presentations. Psychological Monographs, 74(11, Whole No. 498).

SPERLING, G. (1967). Successive approximations to a model for short-term memory, Acta Psychologica, 27, 285-292.

TownSEND, V. M. (1973). Loss of spatial and identity information following a tachistoscopic exposure. Journal of Experimental Psychology, 98, 113-118.

VAN DER HEIJDEN, A. H. C. (1981). Short-term visual information forgetting. London: Routledge \& Kegan Paul.
Van der Heijden, A. H. C., La Hey, W., \& Boer, J. P. A. (1983). Parallel processing of redundant targets in simple visual search tasks. Psychological Research, 45, 235-254.

\section{NOTES}

1. McClelland and Rumelhart's (1981) word-recognition model can serve to elucidate these notions. In their "logogen model," three levels are distinguished: a level with feature detectors, one with letter detectors, and one with word detectors. For present purposes, only the feature level and the letter level are relevant. In their model, feature detectors at the feature level are connected by means of excitatory and inhibitory hardware links with letter detectors at the letter level. A visual input (e.g., an array of letters) excites a number of spatially corresponding feature detectors (for an array of $n$ letters, $n$ spatially separated sets of feature detectors, one set of detectors for each letter presented). Activity at the feature level is automatically and nonselectively translated by means of the hardware connections into specific activity at the letter level. In this model, selective attention can, in principle, intervene at the feature level and at the letter level. In postcategorical filtering, it is assumed that selective attention intervenes at the feature level (i.e., at the input side of the logogens) and that it increases the activity of one set of feature detectors. This operation at the feature level is immediately translated by means of the hardware links in an increased activity of one of the letter detectors at the letter level. In our model, this change in activity at the letter level (i.e., at the output side of the logogen) is identified with selection (cf. Van der Heijden, 1981, chap. VI, for further details). So, postcategorical filtering is early selection (attention operates at the sensory level). But it is also postcategorical selection (identified letters are selected for report). After Sperling (1967, p. 291), it is assumed that the letter detectors are, or are hardware-connected to, "programs of motor instructions."

2. A special problem is posed by the results obtained in Experiment 2. There, subjects followed the optimal strategy, that is, seemed to refuse to report some items appearing elsewhere in the display, although they were not informed that items would not be repeated within a single display. A starting point for an explanation can be found in the basic assumptions underlying postcategorical filtering (cf. Van der Heijden, 1981, pp. 106-110). One of these assumptions is that codes within a domain (e.g., the visual domain or the semantic domain) can be compared, whereas codes in different domains cannot, because they have nothing in common. In the tasks reported in this study, a comparison of codes in store A (i.e., in the visual domain) can show in an early stage of information processing that all colors presented differ. The outcome of such an (automatic) comparison may be at the basis of subjects' reluctance to name a color on the stimulus not indicated by the barmarker. In other words, the information explicitly given in the instruction in Experiment 1 may have become available after a few trials in Experiment 2.

(Manuscript received September 24, 1983; revision accepted for publication April 23, 1984.) 